\title{
Eine neue Imperialismustheorie? Bemerkungen zu dem Buche von Hans-Ulrich Wehler über Bismarcks Imperialismus
}

Der Berliner Politologe und Historiker Hans-Ulrich Wehler hat kürzlich ein umfangreiches Werk vorgelegt ${ }^{1}$, mit dem er, nach dem jahrzehntelangen angeblich erfolglosen *Rätselraten " in der Wissenschaft, den Schlüssel zu Bismardks Überseepolitik gefunden zu haben glaubt. Die bisherigen Erklärungsversuche werden von ihm in bewußter Polemik zumeist entweder in die Kategorie orthodoxer Bismardk-Verehrung und -Apologetik ${ }^{2}$ oder in diejenige eines flachen Vulgär-Marxismus ${ }^{3}$ eingereiht. Mit dem Bewußtsein, ein umfassendes, jedoch eindeutige Prioritäten setzendes Erklärungsmodell - eine "kritische Theorie« - für Bismarcks Imperialismus geboten zu haben, verbindet sich bei Wehler die Fähigkeit, sein Modell wirksam bekannt zu machen: Er hat seine Grundthese innerhalb eines Jahres nicht nur durch eine Zweitauflage seines Buches, sondern auch durch Übersetzung eines umgearbeiteten Kernkapitels ins Englische 4, dessen Rüdkübersetzung ins Deutsche und zweimaligen Wiederabdruds ${ }^{5}$ in Dritt-, ja Viertfassungen (zusammengenommen sechs Fassungen!) publiziert. Das bisherige Echo in der Presse und der Fachwelt ${ }^{6}$

1 Bismardk und der Imperialismus, Köln, Berlin (1969). 1970 folgte eine 2. unveränderte Aufl. (zit. Bismard).

Z. B. G. Ritter, E. Kessel, W. Treue, W. Poels, E. R. Huber.

- Z. B. G. W. F. Hallgarten. Der Passus über die polemische Verwendung seines Begriffssystems: Bismards, S. 38.

4 Bismard's Imperialism 1862-1890, in: Past and Present 48 (1970), S. 119-155.

5 Der in der vorigen Anm. genannte Beitrag ist in deutscher Obersetzung erschienen unter dem Titel: Bismardss Imperialismus 1862-1890, in: Imperialismus. Hrsg. v. H.-U. Wehler, Köln, Berlin (1970) (= Neue Wissenschaftliche Bibliothek 37), S. 259-288 (zit. Imperialismus); ein weiterer Abdrudk unter demselben Titel, in: H.-U. Wehler: Krisenherde des Kaiserreichs 1871 bis 1918. Studien zur deutschen Sozial- und Verfassungsgeschichte, Göttingen (1970), S. 135 bis 161 (zit. Krisenherde). Vgl. auch die Zusammenfassung seiner $*$ kritischen Theorie unter dem Titel: Sozialimperialismus, in: Imperialismus, S. 83-96 (zu diesem Sammelband vgl. die Besprechung von M. Geyer, in: MGM 1/1971, S. 221-230; ferner meine kurze Rezension, in: Historisches Jahrbuch 91 [1971], H. 2). Weitere Abhandlungen Wehlers zum Imperialismus: die Einleitung zum Sammelband Imperialismus, S. 11-36; Wiederabdrudk in: Krisenherde, S. 113-134; ferner: Bismardks späte Rußlandpolitik 1879-1890, ebd., S. 163-190; davon unwesentlich geänderte Neufassung, in: Das kaiserliche Deutschland. Politik und Gesellschaft 1870-1918. Hrsg. v. M. Stürmer, Düsseldorf (1970), S. 235-264. Zur deutschen Sozial- und Wirtschaftsgeschichte im Zeitalter Bismardss vgl. auch den Bericht von M. Stürmer: Nicht Blut und Eisen, sondern Kohle und Stahl ... Bismardss Deutschland im Lidht der Sozial- und Wirtschaftsgeschichte, in: MGM 1/1969, S. 165-177.

- Die ausführlicheren mir bekanntgewordenen Besprechungen: $W$. Schickling, in: Christ und Welt v. 24. 4. 1970, S. 15; W. Treue, in: FAZ v. 11. 7. 1969, S. 10; W. J. Mommsen, in: Die Welt der Literatur v. 8. 10. 1969, S. 58, 60; K. Hildebrand, in: Die Zeit v. 15. 8. 1969; M. Stürmer : Revolutionsfurcht und überseeische Expansion im Zeitalter Bismardks, in: Neue Politische Literatur 15 (1970), S. 188-198; W.-D. Narr: Imperialismus als Innenpolitik, ebd., S. 199-212; H. Herzfeld, in: Historische Zeitschrift 210 (1970), S. 725-728; W. Zorn, in: Vierteljahrsschrift für Sozial- u. Wirtschaftsgeschichte 57 (1970), S. 129-131; N. Rid, in: The Journal of Modern History 42 (1970), S. 421-423; H. Rothfels, in: ders.: Bismards. Vorträge und Abhandlungen, Stuttgart (1970), S. 10-11; H. Seier, in: Sdweizerische Zeitschrift f. Geschichte 20 (1970), S. 422-424; O. Pflanze, in: The American Historical Review 75 (1969/70), S. 1146-1147; G. W. F. Hallgarten: War Bismardk ein Imperialist? Die Außenpolitik des Reichsgründers im Licht der Gegenwart, in: Geschichte in Wissenschaft und Unterricht 22 (1971), S. 257-265; H. G. Hodkerts: Eine neue Theorie des Imperialismus? In: Civitas 10 (1971). 
erlaubt es, das Erscheinen seines Buches mit dem Eklat zu vergleichen (wenn auch nicht gleichzusetzen), den Fritz Fischers Werk über den Ersten Weltkrieg vor einigen Jahren hervorgerufen hat.

In dem umfangreichen Einleitungskapitel seines Buches entwidkelt Wehler eine »kritische Theorie", das heißt eine Arbeitshypothese, des Imperialismus in der zweiten Hälfte des 19. Jahrhunderts. Kernpunkt der Theorie ist die Feststellung, daß der Durchbruch der industriellen Revolution in der okzidentalen Welt wirtschaftliche und soziale Probleme von bis dahin unbekanntem Ausmaß geschaffen habe, vor allem das Problem des ungleidhmäßigen Wachstums der wirtschaftlichen Entwidklung und das Problem der Entstehung eines Massenproletariats am Rande oder außerhalb des überkommenen sozialen Gefüges. Da Wehler von der von Nationalökonomen des 19. Jahrhunderts ausgebildeten Überproduktionstheorie ausgeht, wonach die hochkapitalistische Produktionsweise zu einer Massenherstellung von Wirtschaftsgütern führe, die auf dem Binnenmarkt allein nicht abgesetzt werden könnten und daher nach einem Ausfluß auf den Außenmarkt drängten, und da er sich ferner die von Konjunkturforschern des 20. Jahrhunderts gemachte Entdedkung der Konjunkturschwankungen ${ }^{7}$ zunutze macht, ist seine Theorie eine eklektische Theorie. Neu ist dagegen ihre systematische Anwendung auf Bismardks Überseepolitik zwischen 1862 und 1890. Wehler versucht zunächst den Nachweis zu erbringen, daß unter den Zeitgenossen über die Diagnose der Wachstumsstodkungen Einhelligkeit geherrscht habe. Demgegenuiber tritt die Herausarbeitung des Dissenses über die zur Behebung dieser Stodkungen anzuwendenden Mittel in den Hintergrund. Doch wird deutlich, daß der Warenexport, vor allem in die industriell noch nicht entwidkelten Regionen der Erde, von der Wirtschaft und Geschäftswelt, von Konjunkturtheoretikern, von den Parteien und vom Staat, das heißt in erster Linie von Bismardk, als unabdingbar für die Beseitigung der Schwierigkeiten angesehen wurde.

Im Hauptteil werden sodann die trotz des bis 1879 herrschenden Wirtschaftsliberalismus staatlich geförderte Außenhandelspolitik (Ausweitung des Konsulatswesens, Schaffung von Überseebanken und subventionierten Dampferlinien) Preußens beziehungsweise Preußen-Deutschlands zwischen 1862 und 1879 und der mit der Wende zum Schutzzollsystem zusammenhängende Übergang zum Kolonialerwerb, der 1884/85 in der Besitzergreifung von Kolonien in Afrika und im Pazifik gipfelte, mit den klassischen Methoden der historischen Wissenschaft analysiert. Wehler bietet hier eine Zusammenfassung, die zum erstenmal sämtliches erreichbare gedrudkte und ungedrudkte Material verwertet. In einem Schlußkapitel verbindet Wehler seine "kritische Theorie " mit der Analyse und kommt zu dem Ergebnis, daß die an seine Theorie gestellten Anforderungen der Analyse vollauf entsprächen und er somit eine weinleuchtende, erklärungskräfrige* Theorie von Bismarcks Imperialismus, dem "Sozialimperialismus" erarbeitet habe. Wehlers Werk leidet nicht nur in seinem Hauptergebnis - daß der sozialökonomische Faktor absolut vorrangig vor allen anderen Faktoren, mithin Bismardks Überseepolitik allein unter dem Primat der Innenpolitik adäquat zu verstehen sei - an Einseitigkeit; es stedkt auch voller Widersprüche. Die eingehend ausgearbeitete "kritische Theorie" wird in der folgenden Analyse spatenstichweise untergraben, eine Stütze nach der anderen wird herausgebrochen, und übrig bleibt eine Perspektive, welche die Vielfalt des historischen Lebens in unzulässiger Weise verengt. 
Nach Wehler übte der deutsche Imperialismus unter Bismardk, der letztlich "Sozialimperialismus" gewesen sei, im wesentlichen drei Funktionen aus: 1. In wirtschaftspolitischer Hinsicht sei er ein Bestandteil der staatlich geförderten Außenhandelspolitik und zugleich einer Konjunkturpolitik gewesen, mit deren Hilfe der heranwachsende Interventionsstaat dem expansiven System des Hochkapitalismus Wachstumsstörungen und Absatzschwierigkeiten zu ersparen strebte. Bismarck habe dabei unter der Einsicht in die Gewalt von Sachzwängen - einer ständig expandierenden Wirtschaft - gehandelt. 2. Auf dem Gebiet der Innenpolitik habe der Imperialismus als Integrationsmittel in einem klassenzerrissenen Nationalstaat« gedient. Der 1883/85 hochschäumende Kolonialenthusiasmus in Deutschland sei, wie etwa auch der Antisemitismus oder die Anglophobie jener Jahre, eine Form des Eskapismus gewesen, also der Versuch, aus der "Versumpfung * - ein zeitgenössisches Schlagwort - und der Misere des deutschen Lebens herauszukommen. Bismarck sei 1884 auf der Welle des "Kolonialrausches" geritten, um die Reichstagswahlen des Jahres - ähnlich wie 1879 mit dem "Roten Gespenst « - zu "manipulieren " ${ }^{8}$. 3. Gesellschaftspolitisch habe Bismarck diesen Sozialimperialismus zur Verteidigung der traditionellen Sozial- und Machtstruktur des preußisch-deutschen Staates eingesetzt, zur Erhaltung des gesellschaftlichen status quo, zur "Blockierung " des Emanzipations- und Demokratisierungsprozesses in der industriellen Gesellschaft in Deutschland.

Die Kritik hat an verschiedenen Stellen anzusetzen. Was zunächst den Wert von Wehlers "kritischer Theorie " betrifft, so ist er vorab in einem der von ihm aufgestellten Kriterien äußerst zweifelhaft: Seine Theorie habe ihre Bewährung bestanden, sagt Wehler einmal ${ }^{\theta}$, wenn sie einem Vergleich mit den nichtdeutschen Erscheinungsformen des (Sozial-)Imperialismus standhalte. Wehler ist zugute zu halten, daß er - dadurch einen Kardinalfehler in Fischers erstem Buch vermeidend - bestrebt ist, nicht nur die Probleme der Industrialisierung in gesamteuropäischem Rahmen zu sehen, sondern auch bei der Analyse von Bismarcks Überseepolitik die Konkurrenz unter den europäischen Staaten nicht aus dem Auge zu verlieren. Doch ist es methodisch unzureichend, Bismardks Sozialimperialismus - selbst unter der Voraussetzung, daß man ihn als adäquate Erklärung für die deutschen Verhältnisse akzeptiert - gleichsam zu internationalisieren, indem man anhand einiger Zitatfetzen den englischen, franzö-

Wehlers These von Bismarcks Sozialimperialismus müßte sich am handgreiflichsten an eben diesen Reichstagswahlen erhärten lassen. Man lese einmal sorgfältig den entsprechenden Abschnitt (Bismarck, S. 474-480). Man wird feststellen, 1. daß W.s Wahlanalyse völlig unzureichend ist (die Frage: hat Aussicht auf Kolonialerwerb das Wählerverhalten beeinflußt, bleibt unbeantwortet), 2. daß das Wahlergebnis für die "gouvernementalen « Parteien (Freikonservative, Nationalliberale) nicht gerade ermutigend war (sie schnitten gegenüber 1881 kaum besser ab, 1887 jedoch, ohne Kolonialagitation, weit besser als 1881). Auf solch schwankendem Grund ist W.s These nicht stichhaltiger geworden. Seine Beurteilung des Wahlergebnisses (ebd., S. 479) ist im übrigen ein besonders offenkundiger Beweis für die "Handhabung der Quellen im Sinne seiner These: Von einer "schweren Niederlage « gegenüber 1881 der (kolonialfeindlichen) Linksliberalen kann nur im Hinblids auf die Mandatszahl (106 : 67), nidht die Stimmenzahl (1 078000 : 997000 ) die Rede sein; die (längst nicht durchweg kolonialfreundlichen) Konservativen erhöhten trotz erheblichen Mandatsgewinns (50:78) ihre Stimmen nur unwesentlich ( 831000 : 861000 ; Stimmenanteil 16,3\% : 15,2\%), desgleidhen die (Bismarck-freundlichen) Freikonservativen (379 $000: 388000$; Stimmenanteil 7,4\%:6,8\%); die (stark kolonialfeindlichen, von W. gar nicht erwähnten) Sozialdemokraten verdoppelten ihre Mandatszahl (12: 24); ihre Stimmenzahl stellte diejenige der Freikonservativen in den Schatten (312000:550 000).

- Bismardk, S. 26. 
sischen und amerikanischen Imperialismus ${ }^{10}$ - vom russischen, japanischen, belgischen, italienischen und so weiter ganz zu schweigen - als Ausprägungen eines und desselben Phänomens ansieht. Der Beweis dieser Behauptung setzt eine jeweils eingehende Analyse voraus, die Wehler nicht geliefert hat. Die Ergebnisse der bisherigen umfangreichen internationalen Imperialismusforschung lassen eine Übertragung des Begriffs des deutschen Imperialismus in Form des Sozialimperialismus auf andere Länder nicht oder doch nicht ohne weiteres zu. Ein Meister-Manipulator und Monster-Verschwörer, als den Wehler Bismardk unter Anerkennung seiner zentralen Bedeutung für die deutsche Geschichte seiner Zeit im Grunde sieht, wird sich ohnehin in keinem anderen Land finden lassen. Und das Mischungsverhältnis der Motive, die für die überseeische Expansion jeweils heranzuziehen sind, ist so unterschiedlich, daß eine generalisierende Formel eine Verkürzung der historischen Vielfalt bedeuten würde.

Von überragender Bedeutung für Wehlers These ist sein Imperialismusbegriff. Er ist einerseits zu weit gefaßt und läßt sich daher fast beliebig aufblähen, anderseits ist er $\mathbf{z u}$ eng und einseitig. Wehler definiert Imperialismus als »diejenige direkte-formelle und indirekte-informelle Herrschaft..., welche die okzidentalen Industriestaaten unter dem Druck der Industrialisierung mit ihren spezifischen ökonomischen, sozialen und politischen Problemen und dank ihrer vielseitigen Überlegenheit über die weniger entwidkelten Regionen der Erde ausgebreitet haben " 11.

Zunächst einmal erscheint es uns gefährlich - da es einer Verflüchtigung des Begriffsinhaltes Tür und Tor öffnet -, den von der englischen Imperialismusforschung an der einzigartigen Erscheinung des mittelviktorianischen Empire entwickelten, heute noch umstrittenen Begriff des "informal empire ohne weiteres auf nichtenglische Verhältnisse zu übertragen. Als (Handels-)Imperialismus wäre dann jede Art wirtschaftlicher Expansion eines Industriestaates zu bezeichnen, etwa die Politik der "Offenen Tür" der USA in Japan und China seit 1854 oder die Außenhandelsvertragspolitik Caprivis nach 1890. Da Wehler Imperialismus grundsätzlich als auch nach dem Ersten Weltkrieg (mindestens bis

10 Ebd., S. 116-119. - Die dort wiedergegebenen Zitate sind wieder eines der Beispiele für den Umgang W.s mit den Quellen. W. wird wissen, daß die französische Imperialismusforschung die öffentlichen Außerungen J. Ferrys, in denen ökonomische oder sozialökonomische Motive für den Kolonialerwerb Frankreichs anklingen, nicht prima facie nimmt; solde Motive haben vielmehr nur als Rechtfertigung für den essentiell politischen Charakter des französischen Imperialismus (Rehabilitationsbedürfnis nach der Niederlage von 1870/71) gedient. - Die den englischen Sozialimperialismus angeblich beweisenden Quellen sind im übrigen äußerst dürftig. Einschlägige Āußerungen des neuseeländischen (!) Premierministers und C. Rhodes' (!) reichen für einen Nachweis überhaupt nicht aus. Die entscheidenden Politiker (Salisbury, Rosebery, Chamberlain) haben natürlidh nicht "sozialimperialistisch argumentiert. Während der zweiten Wirtschaftskrise nach 1882/83 war in England bezeidnenderweise das expansionsfeindliche Ministerium Gladstone (ein »Little Englander a) an der Macht. W. läuft mit seiner unbewiesenen These sdhnurstradks den Ergebnissen der englischen Imperialismusforschung zuwider. In seiner Polemik gegen die englischen Forscher D. K. Fieldhouse, R. E. Robinson u. J. Gallagher (Imperialismus, S. 17; Krisenherde, S. 120) verläßt W. im übrigen seine industrialisierungsbezogene Imperialismustheorie. $\mathrm{DaB}$ hinter der politisch motivierten Besetzung Agyptens der Wirtschaftsfaktor Indien stand, steht außer Zweifel, hat aber mit Wehlers Imperialismusbegriff nichts zu tun. Das indische Kolonialreich ist vor der Industrialisierung gegründet worden. Und die Besetzung Agyptens läßt sich nur aus der Kontinuität der britischen Reichsbildung verstehen. Auch im Falle der USA wird von der Mehrheit der Sachkenner, soweit ich sehe, die These abgelehnt, daß die USA neuer Absatzmärkte bedurft hätten, um mit den Problemen der Uberproduktion fertig zu werden. Der amerikanische Imperialismus wird vielmehr aus anderen Motiven hergeleitet: natïrliches Expansionsbedürfnis nach dem Fortfall der *Frontier*, Missionierungsgedanken, Einfluß der Lehren des Marineschriftstellers A. Mahan bezüglich einer starken Marine, Neubesinnung auf die Monroe-Doktrin.

11 Bismardk, S. 23; ähnliah Imperialismus, S. 11 u. Krisenherde, S. 113. 
1945) existent voraussetzt und Hitlers Ostlandritt expressis verbis in die Kontinuität des deutschen Imperialismus seit Bismardk eingebettet sieht ${ }^{12}$, besteht keine Notwendigkeit, das wirtschaftliche Eindringen Chinas von heute in den afrikanischen Kontinent (Bahnbau in Tanzania) nicht als Imperialismus zu bezeichnen. Erst recht müßte die Herrschaft der Sowjetunion über Ostmitteleuropa als Imperialismus aufzufassen sein, da hier nicht nur informeller Wirtschaftsimperialismus, sondern direkte territoriale Herrschaft (Stationierung von Truppen, die "sozialimperialistisch", das heißt zur Aufrechterhaltung der sowjetischen Macht eingesetzt werden) ausgeübt wird. Bezeichnenderweise wird dieser Vergleich nie, auch nur andeutungsweise, gezogen, wohl nicht zuletzt deshalb, weil Wehler bei seiner "kritischen Theorie " von marxistischen Prämissen ${ }^{13}$ ausgeht. Es besteht ferner keine Denknotwendigkeit, die Herrschaft (= Abhängigkeitsverhältnis) eines Staates über nur einen Wirtschaftssektor oder wenige Wirtschaftssektoren eines anderen nicht Imperialismus zu nennen. Folglich kann gesagt werden, daß die okzidentalen Staaten nicht nur im China des 19. Jahrhunderts imperialistisch vorgingen, sondern auch Frankreich in Rußland (durch Kapitalinvestitionen im Eisenbahnbau besonders seit 1890) oder Deutschland in Europa (durch Beherrschung der elektrotechnischen und chemischen Produktion) Imperialismus trieben; das heißt auch die imperialistischen Staaten können ihre Beziehungen untereinander imperialistisch gestalten, sofern ein wirtschaftliches Abhängigkeitsverhältnis entsteht. Ja, es ist sogar der Fall denkbar, daß ein nichtokzidentales Land über ein okzidentales wegen seiner wirtschaftlichen Monopolstellung Imperialismus ausübt (das Scheichtum Kuweit wegen seiner Beherrschung des englischen Erdölmarktes). Ein weiterer Gesichtspunkt: Es liegt kein logischer Zwang vor, Wehlers Definition nicht auch dahingehend zu modifizieren, (Handels-)Imperialismus auch vor dem Zeitalter der Industrialisierung anzusetzen. Das klassische Beispiel wäre das England des 18. Jahrhunderts, das seit Utrecht 1713 erst Spanien, dann Frankreich in Übersee wirtschaftlich aus dem Felde schlug und ein riesiges Kolonialreich beherrschte. Die soziale Imperialismuskomponente fällt bei folgendem Vorgang ins Auge: In der ersten Hälfte des 17. Jahrhunderts gab es in den Niederlanden, vor allem in Kreisen um die Westindische Kompanie, Überlegungen, für die aus dem spanischen Flandern vertriebenen Kalvinisten eine neue Heimat in Übersee zu schaffen ${ }^{14}$.

Man erkennt an diesen wenigen bis zur Absurdität getriebenen Beispielen die beliebige Austauschbarkeit und damit den zunehmenden Verlust an Präzision eines solchen Imperialismusbegriffes. Auf die andererseits zu beobachtende Einseitigkeit wird noch zurüdszukommen sein.

Einseitig und daher problematisch erscheint uns auch Wehlers Bewertung der wirtschaftlichen Trendperiode von 1873 und $1896 \mathrm{zu}$ sein. Er orientiert sich zu eng an dem Vokabular der zeitgenössischen Quellen und der ihr folgenden Forschung. Der (von England herrührende) auf die genannte Trendperiode angewandte Begriff der "Großen Depression" vermittelt heute jedoch ein unhistorisch einfarbiges Bild, weil er unverwischbar von dem durch und durch schwarzen Bild der Weltwirtschaftskrise von 1929 gefärbt erscheint. Wehler

12 Bismard, S. 501; Imperialismus, S. 283; Krisenherde, S. 161.

18 Vgl. z. B. Bismards, S. 29 (besonders Anm. 19), S. 112. Dagegen wird von der vermeintlichen Existenz eines amerikanischen (informellen) Imperialismus in der beutigen Zeit direkt gesprochen (Imperialismus, S. 29; Krisenherde, S. 133). Sold durchsichtige Propaganda kennzeidhnet besonders den Sammelband über den Imperialismus.

14 (Sozial-)Imperialismus reinsten Wassers wurde (vor der Industrialisierung) bei der russischen Eroberung Sibiriens und der englischen Aneignung Australiens getrieben: beide Gebiete waren ursprünglich Strafkolonien. 
selbst zieht letztere - vor allem als methodologische Rechtfertigung für den Ausgangspunkt seiner "kritischen Theorie " 15 - für sein Vorgehen auch direkt heran. Doch für die Ausbildung des Imperialismusbegriffs wichtiger als die Feststellung der Gemeinsamkeiten beider Phänomene ist die Betonung ihrer Unterschiede. So hat Wehler es vermieden, von seinem Vorbild Hans Rosenberg ${ }^{16}$ die grundlegende (ihrerseits auf zeitgenössische Quellen zurückgehende) Einsicht zu übernehmen, daß die Wirtschaftskrise nach 1873 in erster Linie eine Krise der reichen und nicht - wie auch 1929ff. mit der Massenarbeitslosigkeit - der armen Leute gewesen ist; daß sich nicht nur (entgegen der Marxschen Unterkonsumtionsthese) die Nominal-, sondern auch die Reallöhne der Arbeiter kontinuierlich verbessert haben; daß mithin die zunehmende Unruhe unter den Arbeitern nicht aus ihrer ökonomischen Verschlechterung (entsprechend der Marzschen Verelendungstheorie), sondern aus einer materiellen Verbesserung ableiten läßt eine Erscheinung, die uns in unserer heutigen Zeit psychologisch durchaus verständlich ist. Aus dem gleichen Grunde scheint uns auch die Verwendung von Begriffen wie Wachstumsstörungen, Schodkwirkungen, Trauma etc. geeignet, den Gesamteindrudk - im Gegensatz wieder zu 1929 - zu verfälschen. Ursache und Wirkung werden nur zu leicht ins Gegenteil verkehrt. Denn die eigentliche Störung war die Konjunkturüberhitzung vor 1873, die sogenannte Krise danach war, aufs ganze gesehen, gar kein Abknicken, sondern ein Abflachen der Konjunkturkurve. Es ist daher von diesem Standpunkt aus gerechtfertigt, die Wirtschafts»krise" als Selbstreinigung und -gesundung eines überstürzt angelaufenen Prozesses zu sehen. Die "Große Depression* erweist sich somit als ein Mythos, denn an der langfristigen Aufwärtsbewegung des Gesamtumfangs der Industrieproduktion ist heute kein Zweifel mehr. In dieser Sicht erscheint dann der ganze Komplex des "Sozialimperialismus« in einem anderen Lichte; er reicht für eine Erklärung des Kolonialrausches in Deutschland 1883/85 iberhaupt nicht aus. Die in Bismardks "Herrschaftstechnik « angeblich dominierende sozialpolitische Funktion des Imperialismus läßt sich nicht ohne weiteres dadurch erweisen und erhärten, daß - wie es Wehler in dem analytischen Teil des Buches in bestechender Beherrschung der Quellenmassen tut - legionenhaft zeitgenössische Quellen zusammengetragen werden - von W. Liebknechts Wort vom Export der sozialen Frage zu Bismardks eigenen ähnlich lautenden bildhaften Außerungen über das zu eng gewordene Kleid der Nation und so weiter. Wehlers Postulat von der Dominanz des sozialökonomischen Faktors ist von den Grundpositionen aus zu verstehen, von denen er ausgeht, aber auch von den Grundpositionen, von denen er nicht ausgeht. Aufschlußreicher als die ersteren, mit deren Andeutung oben es sein Bewenden haben soll ${ }^{17}$, sind die letzteren. Wehler geht von einer falschen oder doch einseitigen Vorstellung des Konservativismus aus. Daß das Bild des Statischen, ja die Vorstellung vom Utopischen ${ }^{18}$ auf den englischen Konservativismus des 19. Jahrhunderts überhaupt nicht zutreffen, wird Wehler

1s Wehler fragt (Bismard, S. 32, Anm. 23): Wenn man die Weltwirtschaftskrise von 1929 und die ihr folgenden Depressionsjahre als von grundlegender Bedeutung für die Geschichte der 1930er Jahre allgemein anerkannt habe, warum solle man dann nicht auf gleiche Weise die Wirtschaftskrisen des 19. Jahrhunderts als Fixpunkt für die Geschichtsschreibung nehmen? Daher wohl auch seine grob simplifizierende These von der Kontinuität des Imperialismus von Bismardk zu Hitler.

16 Große Depression und Bismarckzeit. Wirtschaftablauf, Gesellschaft und Politik in Mitteleuropa, Berlin 1967, S. 43, 47, 87 (= Veröffentlichungen der Historischen Kommission zu Berlin beim Friedrich-Meinecke-Institut der Freien Universität Berlin, Bd 24).

17 Vgl. Anm. 13.

18 Wehler gebraudht mitunter Wendungen wie *konservative Utopie«, Utopie der Status-QuoVerteidigung ( (z. B. Bismardk, S. 115; Imperialismus, S. 27, 40, 283; Krisenherde, S. 131). 
wohl zugeben. Aber auch auf Bismarcks Konservativismus sind sie nicht anwendbar. Allein schon der Pragmatismus, den Wehler im Zusammenhang mit Bismarcks Uberseepolitik eindringlich schildert, ist damit nicht vereinbar, ebensowenig die von ihm herausgearbeitete Einsicht Bismarcks in Sachzwänge der modernen kapitalistischen Wirtschaftsverfassung. Es kann auch nicht von der Konservierung des gesellschaftlichen status quo gesprochen werden, wenn Bismards - wie Wehler mehrfach ${ }^{10}$ feststellt - gefordert habe, ${ }^{\prime}$ daß man realisiert, was in den sozialistischen Forderungen als berechtigt erscheint «. Daß Bismarck den Anruf der sozialen Frage seiner Zeit verstanden hat, beweisen solche Worte ebenso wie das gesamte Werk seiner Sozialpolitik. Daß dieses Werk in einer Sadkgasse gelandet ist, steht auf einem anderen Blatt. Ist es nicht kennzeichnend für den hohen Grad sozialer Mobilität Preußen-Deutschlands und seiner Fähigkeit zu sozialer Integration, daß die mkleine Führungsschicht von Unternehmern *, die nach Wehler ${ }^{20}$ "auf der Kommandobrücke der deutschen Wirtschaft* standen, zu einem maßgeblichen Teil aus dem Arbeiterstand und dem Mittelstand herkam ${ }^{21}$ - was er nicht erwähnt -; daß sich dieser bedeutende Anteil so rasch in die $*$ feudalisierte Gesellschafthierarchie" mit ihren sautoritären Verhaltens- und Denkformen * integrierte? Schließlich ist es für den Konservativismus Bismarcks - ebenso wie auch für seine Vorstellungen von "Imperialismus", den Wehler ja auf die Zeit nach dem Durchbruch der Industrialisierung eingrenzen will - nicht unerheblich, wie stark er im Historischen, das heißt im Bewußtsein historischer Kontinuität verwurzelt war. Immer wieder weist Wehler zu Recht - aber gegen den Kern seiner Imperialismustheorie - darauf hin, wie sich Bismards in seiner Kolonialpolitik an dem historischen Beispiel englischer Überseeherrschaft orientierte und wie er seine Skepsis gegenüber der Errichtung überseeischer Gebietsherrschaft auf historische Erfahrungen der (vorindustriellen) englischen und französischen Kolonialpolitik gründete. Im übrigen widerspricht es einer banalen Einsicht in das menschliche Zusammenleben, in Bismarcks konservativer "Interessenpolitik", die Wehler durch die (Wieder-)Entdeckung seines "Sozialimperialismus« zu entschleiern meint, ein Spezifikum seines Imperialismus oder des klassischen Zeitalters des Imperialismus überhaupt zu sehen. Das Bedürfnis nach Konservierung und Legitimation von Herrschaft ist eine Grundgegebenheit der gesamten Menschheitsgeschichte ${ }^{22}$.

Zwei gewichtige Ingredienzen des okzidentalen Imperialismus im Zeitalter Bismarcks schätzt Wehler, da er starr vom Primat des Sozialökonomischen ausgeht, zu gering ein beziehungsweise vernachlässigt er in seiner Deutung: die internationale Mächterivalität und die psychologische Grundstimmung der Zeit, das heißt den Zeitgeist. Das Gewicht des machtpolitischen Faktors in Bismardks Uberseepolitik, die wir mit Wehler nicht auf deren sichtbaren Höhepunkt $1884 / 85$ begrenzen wollen, ist in seinem analytischen Teil so stark, daß von einer

19 Z. B. Bismardk, S. 187.

20 Ebd., S. 97.

21 Um 1840 waren etwa $61 \%$ der Eltern bedeutender Unternehmer Arbeiter und Kleinbürger, $28 \%$ Angehörige des Mittelstands (selbständige Handwerker, Kaufleute, mittlere Beamte), $11 \%$ Adlige, Geistliche, höhere Beamte usw. Bis 1890 hatte sich das Verhältnis auf die Relation $21: 65: 14$ verschoben. Vgl. W. Treue: Gesellschaft, Wirtschaft und Technik Deutschlands im 19. Jahrhundert, in: [B.] Gebhardt: Handbuch der deutschen Geschichte. Hrsg. v. H. Grundmann, Bd 3, Stuttgart, 9. Aufl. 1970, S. 499.

22 W. gibt diese Banalität zu, wenn er etwa auf die von Machiavelli beschriebenen Herrschaftstechniken (Bismard, S. 115; Imperialismus, S. 27; Krisenherde, S. 131) hinweist, macht sie aber zum Stützpfeiler seiner These von Bismardss - durch die Neuartigkeit der in der Industriellen Revolution geschaffenen sozialen Probleme gekennzeichnetem - „Sozialimperialismusk. 
Unterordnung unter die Innenpolitik schlechterdings nicht die Rede sein kann. Der Versuch einer Stufenordnung mag von heuristischem Wert sein, der historischen Wirklichkeit entspricht sie zumeist nicht. Eher ließe sich das Bild der Medaille mit den wesensgemäß zusammengehörigen zwei Seiten Außenpolitik und Innenpolitik anwenden, wobei einmal die eine, ein andermal die andere Seite mehr zum Vorschein kommt. Die Dominanz, das heißt das Hervorscheinen des Politischen ist beim deutschen Kolonialerwerb 1884/85 nicht zu bestreiten, ebensowenig sind natürlich die von Wehler herausgearbeiteten starken hintergründigen Einflüsse der seit 1882/83 einsetzenden Stagnationsphase der Wirtschaft zu verkennen: Sie sind aber nur die Kehrseite oder, um mit P. Renouvin zu reden, sie stellen die "forces profondes « dar. Die politischen Vorgänge jener Jahre: die englisch-französische Rivalität über Ägypten (Herbert v. Bismardk: der "Zankapfel Agypten" sei »ein Geschenk des Himmels") wie der drohende Zusammenstoß Englands mit Rußland in Zentralasien, sie erst schufen die fast ideale Gunst des Augenblidks und die weltpolitische Handlungsfreiheic in der Bismardk zu direktem Kolonialerwerb sowohl als Mittel (in der Innenpolitik) als auch als Ziel (Kolonialentente mit Frankreich und Erpressung Englands zur Ausbalancierung des Mächtekonzerts und größtmöglicher Erhaltung der Ruhelage des europäischen Kontinents) schritt. $\mathrm{Daß}$ sich das (innenpolitische = sozialpolitische) Mittel rasch als untauglich erwies, zeigen am deutlichsten die schon seit 1886 sich immer mehr verstärkende Skepsis Bismardks über den ökonomischen Wert der Kolonien und die Millionen deutscher Bürger ${ }^{23}$ (darunter natürlich auch wkatalinarische Existenzen «), die durch ihre Auswanderung nach Amerika mit den Füßen gegen die Agitation der Expansions- und Kolonialenthusiasten votierten. Derart rasch wurden die 1884/85 erworbenen Kolonien als das denkbar ungünstigste Objekt für einen "Sozialimperialismus «, das heißt als die denkbar schlechtesten Abzugskanäle sowohl für die deutsche Überproduktion als auch für die sozial unruhigen Elemente erkannt ${ }^{24}$, daß Bismarck in den Folgejahren trotz wachsender innerpolitischer Schwierigkeiten nicht nur nicht an weiteren Kolonialerwerb dachte ${ }^{25}$, sondern vielmehr daran, wie er die den Reichshaushalt belastenden "Sumpfkolonien* und *Sandlöcher* wieder loswerden könnte. Der von ihm noch vorbereitete Sansibar-Helgoland-Vertrag, der in den Augen der deutschen Kolonialenthusiasten "zwei Königreiche" (Uganda, Witu, Sansibar) für eine "Badewanne" hergab, zeigt mit am augenfälligsten die Bedeutung nichtökonomischer, das heißt politischer (und in diesem Fall auch strategischer) Gesichtspunkte, mit anderen Worten die sein ganzes Denken und

:o Während der Trendperiode 1873-1896 fast 2,5 Mill.; am Ende dieser Periode (1897) lebten in den deutschen Kolonien nur über 3000 Deutsche.

24 W.s Grundthese, daß Imperialismus ein Korrelat des Strebens nach Einfrieren des gesellschaftlichen status quo gewesen sei, ist weder theoretisch $\mathbf{z w i n g e n d , ~ n o h ~ s c h e i n t ~ e s ~ h i s t o r i s c h - e m p i - ~}$ rish stringent nachweisbar zu sein. Imperialismus ist in diesen Fällen (in der Stagnationsphase 1883 ff.) zunächst einmal schlicht ökonomisch bedingt: Durch den wachsenden Protektionismus der Industriestaaten wurden ihrem industriellen Export zunehmend Fesseln angelegt. Kapitalexport diente dann häufig dazu, diese Fesseln zu lodkern, indem die Gewährung von Anleihen von Handelskonzessionen seitens des Empfängerlandes abhängig gemacht wurde. Protektionismus führte ferner zu neomerkantilistischen Tendenzen (Autarkiebestrebungen), in denen Kolonien einen natürlichen Platz fanden. Er war schließlich mitverantwortlich für die wachsende Mächterivalität im Zuge der überseeischen Expansion. Trotzdem wird man an der Tatsache nicht vorbeisehen können, daß von den Staatsmännern wirtschaftliche Argumente häufig nur als Rationalisierung ex post facto für ihren Kolonialerwerb verwendet wurden.

23 Auf C. Peters' "Erwerbungen* an ostafrikanischen Steppen und Urwäldern z. B. hätten sich genügend Ansprüche gründen lassen. Doh für Bismards war - zur Erhaltung des europäischen Friedens - der Ausgleich mit England wichtiger (1887 Mittelmeerentente, 1890 SansibarHelgoland-Vertrag) als höchst fragwürdige "sozialimperialistische* Abenteuer. 
Handeln beherrschende Sorge um Erhaltung des Friedens in Europa; Kolonien dienten $\mathrm{ihm}$ als zusätzliches Tauschobjekt in seinem politisch-diplomatischen Instrumentarium ${ }^{26}$. Ohne den Faktor der sich nach 1884/85 und auch noch nach Bismarcks Abgang rasch steigernden Rivalität unter den europäischen Mächten, das heißt ohne den machtpolitischen Aspekt ist die zumeist jedem kaufmännischen Kalkül spottende Ausbreitung Europas über die letzten Sandund Eiswüsten der Erde, über die letzten undurchdringlichen Urwälder und die winzigsten Korallenriffs nicht zu verstehen.

Wir berühren damit auch noch einen letzten widhtigen Gesichtspunkt, den Wehler zwar nicht unerwähnt läßt, über dessen Bedeutung für den deutschen beziehungsweise europäischen Imperialismus er sich jedoch nicht ausläßt. Er gehört zu den "forces profondes« des Phänomens, den wir mit dem allgemeinen Wort "Zeitgeist* umschreiben wollen. Wehler bezeichnet den •Kolonialenthusiasmus «, der ein hervorstechender Ausschnitt aus dem Zeitgeist darstellt, als "Krisenideologie " ${ }^{27}$, verengt seine Herkunft jedoch in seiner marxistischdeterministischen Weise wiederum auf die soziale und ökonomische Misere der ihn tragenden Schichten, das heißt der mittelständischen Gruppen. Der Kolonialenthusiasmus ist für ihn nichts anderes als das Ventil zur Uberwindung von "Frustrationen «. Wehler verfällt hier in den Fehler, den er so manchem bisherigen Imperialismusforscher vorwirft: daß er eine "Theorie « als selbstverständlich gegeben voraussetze, ohne sie empirisch zu erhärten. Gewiß gerät man bei dem Phänomen des "Kolonialrausches in ein Gebiet, in dem man anders als auf dem Felde der Wirtschaft an die Grenze des exakt Nachweisbaren und Wägbaren stößt. Die Schwierigkeiten des methodischen Vorgehens entheben den Forscher jedoch nicht der Notwendigkeit, die Einzelelemente jenes Phänomens möglichst umfassend zu analysieren. Eines seiner wichtigsten Elemente ist zweifellos der Sozialdarwinismus, der als "Krisenideologie" im Wehlerschen Sinne einfach nicht abzutun ist. Er war eine Popularphilosophie von heute schwer faßbarem Einfluß, von dem sich ohne große Mühe eine Brücke zum Militarismus schlagen ließe ${ }^{28}$. Ihn gilt es einmal im politischen Entscheidungsprozeß umfassend nachzuweisen ${ }^{29}$. Nicht nur ganze Wissenschaftszweige waren damals von ihm beherrscht (wie die Soziologie); der Machtkampf der Gruppen ("Kampf ums Dasein «, "Überleben des Stärkeren «) wurde vielmehr allgemein als ein Grundgesetz der Menschheitsgeschichte angesehen. Seine Verbindung zum Imperialismus ist sofort einsichtig: Er rechtfertigte die rüdksichtslose Behandlung der *niederen Rassen« und die Nichtachtung oder Zerstörung der in den Kolonien

98 Deutsch-Südwestafrika mußte bereits 1889, Deutsch-Ostafrika 1891 vollständig in Reichshand übergehen; d. h. das kaufmännische Kalkül der (Charter-)Gesellschaften hatte sich rasch als Fehlrechnung erwiesen (ihr einziger finanzieller Erfolg war die vom Staat gezahlte Entschädigungssumme). Der Staat trieb jetzt also mit offensidhtlich defizitären Kolonien imperialistische Politik - gewiß nicht aus ökonomischen Motiven.

I7 Vgl. etwa Imperialismus, S. 27; Krisenherde, S. 132.

28 Vgl. dazu G. Ritter: Staatskunst und Kriegshandwerk. Das Problem des Militarismus« in Deutschland. Bd 2: Die Hauptmächte Europas und das wilhelminische Reich (1890-1914), München, 2. Aufl. 1965, S. 135-147. Ders.: Das deutsche Problem. Grundfragen deutshen Staatslebens gestern und heute, München, 2. Aufl. 1966, S. 108-146.

2y Der Ritter-Schüler H.-G. Zmarzlik sagt m. E. zu Recht, daß der Sozialdarwinismus politisch mmassive Wirkungen $\propto$ hatte, in: Wieviel Zukunft hat unsere Vergangenheit? Aufsätze und Uberlegungen eines Historikers vom Jahrgang 1922, München (1970), S. 10. Vgl. ebd., S. 56 bis 85 seinen Aufsatz: Der Sozialdarwinismus in Deutschland als geschichtliches Problem. Ebd., S. 265-266, die relativ spärliche Literatur der letzten Jahre. Vgl. auch meinen Aufsatz: Zur Theorie des Imperialismus, in: Aus Politik und Zeitgeschichte, Beil. zur Wochenzeitung - Das Parlament «, B 23/71 (5. Juni 1971), S. 3-11, in dem ich in anderem Zusammenhang bereits einige der hier niedergelegten Ansichten vorgetragen habe. 
vorgefundenen andersartigen Kultur- und Sozialformen. Daß er nicht ohne Einfluß auf den handelnden Politiker (besonders in England) war, zeigt das Beispiel Salisburys, der von den "dying nations " sprach; zeigt schließlich die Generation der Wilhelms II., Th. Roosevelts, Chamberlains, Leopolds II. und so weiter. Selbstverständlich wurde der Sozialdarwinismus (besonders in Deutschland) auch als herrschend in den Beziehungen der europäischen Völker untereinander anerkannt und erklärt daher zu einem guten Teil die Ubersteigerung des Nationalismus und des Prestigebedürfnisses (vergleiche den Sport oder, wenn man will, den Kult des Flaggenhissens - damals auf irgendeinem Südsee-Atoll oder in einer Wüsten-Oase, heute in einem Mondkrater) der einzelnen Kolonialmächte. Schließlich gehören in diesen Zusammenhang auch Urtriebe des Menschen, wie Forschergeist, Eroberungsdrang, Abenteurerlust ${ }^{30}$ der Kolonialpioniere (Livingstone, Stanley, de Brazza und andere), die unabdingbare Voraussetzungen für den okzidentalen Imperialismus gewesen sind, mit "Sozialimperialimus a aber kaum etwas zu tun haben. Wehler widmet dem Imperialismus treibenden Konquistador Carl Peters einen ausführlichen Abschnitt ${ }^{31}$, in dem er zu Recht das pathologische Geltungsbedürfnis dieses Mannes sehr hoch, das durchaus vorhandene wirtschaftliche Motiv jedoch gering veranschlagt. Nur mit den mächtigen Geistesströmungen der Zeit lassen sich sein Einfluß auf das öffentliche Denken in Deutschland, läßt sich die Megalomanie des "Empirebuilders " Cecil Rhodes, das Zittern einer ganzen Nation um den totgeglaubten Emin Pascha (Eduard Schnitzer) oder die schrillen Töne der "gelben Presse nach der Tragödie Gordons vor Khartum hinreichend erklären. Die Eroberung der meisten nach 1880 noch nicht beherrschten Regionen der Erde bis hin zum Südpolargebiet (und heute die Eroberung des Mondes, läßt man die militärischen Gesichtspunkte einmal aus dem Spiel) ist in erster Linie durch national- und sozialpsychologische Faktoren, wie Prestigesucht, Rivalität, elementarer Forscherdrang auszudeuten. Die Imperialismusforschung steht auf diesem Gebiet vor einem noch weithin unbeackerten Felde.

Es ist nicht das erste $\mathrm{Mal}$ in der jüngsten Geschichte der Historie, daß einer ihrer Angehörigen den Mut aufgebracht hat, in einem großen Wurf zu versuchen, die bisher gültigen Einsichten und Erkenntnisse radikal in Frage zu stellen und sie durch neue zu ersetzen. Der Wurf scheint uns nicht gelungen, nicht eigentlich weil Wehler zahlreiche Lüdken in unserem Wissen nicht ausgefüllt hat ${ }^{32}$ - das kann nur durch eine Vielzahl von Einzelstudien geleistet werden -, sondern weil er die von ihm analysierten Faktoren des Okonomischen und des Sozialen als alle anderen Motive beherrschend verabsolutiert und sich

so Daß solche Motive »dem Historiker schwerlich weiterhelfen « (Imperialismus, S. 12; Krisenherde, S. 115), bedeutet nicht, daß sie nicht existierten.

i) Bismardk, S. 333-372.

s2 Als Desiderate der Forschung fallen (abgesehen von dem Riesenkomplex des "Zeitgeistes๔: der englische »Jingoism» als Antriebskraft des Imperialismus; das Schulwesen, die Universität, der Geschichtsunterricht, die Literatur, die Kirche im Dienste oder als Antriebskraft des Imperialismus etc., etc.) u. a. auf: eine umfassende Analyse des "Deutshen Kolonialvereins* und der \#Gesellschaft für Deutsche Kolonisation*; das Britische Empire als Vorbild für das koloniale Denken und für die Kolonialbewegung in Deutschland; Untersuchungen der Reichstagswahlen der Bismardszeit; eine Peters-Biographie (überhaupt einschlägige biographische Forschung); die Bedeutung der Konsularberichterstattung für die politische Willensbildung; Bismardks Agyptenpolitik; anti-imperialistische Strömungen in Deutschland (besonders die Haltung der Liberalen zur Oberseepolitik); Arbeiterschaft und Imperialismus in Deutschland, in England. - Uber die Ergebnisse der jüngsten (west-)deutschen Forschung über die deutsche Kolonialherrschaft vgl. meinen Bericht: Die deutsche Kolonialherrschaft in Afrika. Neue Wege der Forschung, in: Vierteljahrsschrift für Sozial- u. Wirtschaftsgeschichte 58 (1971), S. $468-481$. 
so den Weg zu einer umfassenderen Zusammenschau verbaut hat. Nur durch Anerkennung der realiter unauflöslichen Interdependenz sämtlicher hier angedeuteten Motive wird einmal eine allumfassende Imperialismustheorie gefunden werden können. Je niedriger bei künftigen einschlägigen Forschungen der Anspruch angesetzt wird, um so besser für die Anerkennung der erbrachten Leistung. Wehler scheint uns den selbstgesetzten Anspruch nicht erfüllt zu haben, doch wird sein Buch hoffentlich eine Welle neuer Detailforschungen in Gang bringen. Das dürfte wohl nicht zuletzt die Absicht Wehlers gewesen sein, die unausgesprochen hinter seinen Imperialismusforschungen steht. Er hat die Entwicklung zu der Einsicht, zu der sich ein ihm so geistesverwandter Imperialismusforscher wie J. Hobson schließlich bekannt hat, daß nämlich die Auffassung von der ökonomischen Determiniertheit der Geschichte diese in ein Prokrustesbett zwängt, noch nicht durchgemacht. Jeder Forscher, der Imperialismus ausschließlich oder vorrangig in einem ökonomischen oder sozialökonomischen Koordinatensystem definiert, ist ein Rationalist, der glaubt, eine rationale Erklärung für das Verhalten anderer, das heißt für das Verhalten der europäischen Völker beim Aus- oder Aufbau ihrer Kolonialreiche, die weniger planmäßig als improvisiert entstanden, gefunden zu haben. Nichts widerspricht der Macht der Idee in der Geschichte und der menschlichem Leben innewohnenden Irrationalität mehr als dieser Fehlschluß. Lenin, selbst ein Rationalist (auch in der Suche nach den Motiven des Imperialismus), dem sich Wehler als einem seiner Lehrmeister verpflichtet fühlt ${ }^{33}$, lebte diesen Fehlschluß in grandioser Weise vor, indem er Künder einer zündenden Idee und einer der größten Machtmenschen wurde, den die Geschichte je gekannt hat. 
